\section{Low Pathogenicity Avian Influenza (H5N2) Viruses, Dominican Republic}

David H. Chung, Dejelia R. Gomez, Julia M. Vargas, Belkis L. Amador, Mia K. Torchetti, Mary L. Killian, David E. Swayne, Dong-Hun Lee

\begin{abstract}
Author affiliations: University of Connecticut, Storrs, Connecticut, USA (D.H. Chung, D.-H. Lee); Ministry of Agriculture, Roseau, Dominican Republic (D.R. Gomez, J.M. Vargas, B.L. Amador); US Department of Agriculture, Athens, Georgia, USA (D.E. Swayne); US Department of Agriculture, Ames, lowa, USA (M.K. Torchetti, M.L. Killian)
\end{abstract}

DOI: https://doi.org/10.3201/eid2612.200268

Low pathogenicity avian influenza (H5N2) virus was detected in poultry in the Dominican Republic in 2007 and re-emerged in 2017. Whole-genome sequencing and phylogenetic analysis show introduction of an H5N2 virus lineage from Mexico into poultry in the Dominican Republic, then divergence into 3 distinct genetic subgroups during 2007-2019.

L ow pathogenicity avian influenza virus (LPAIV) subtype H5N2 has caused outbreaks in poultry in Mexico since 1993 and mutated into highly pathogenic avian influenza virus (HPAIV) H5N2 during 1994-1995 (1). In 1994, a vaccination program against H5N2 in poultry was established in Mexico; HPAIV H5N2 was eradicated there in 1995 (2). However, LPAIV H5N2 persisted and related viruses spread to neighboring countries $(1,3,4)$. In addition, the H5N2 virus lineage from Mexico was introduced to Taiwan in 2003, likely because of inadequately inactivated vaccines. The virus then reassorted with the local avian influenza (H6N1) virus strain that has been enzootic in chickens in Taiwan since 1997 to produce reassortant $\mathrm{H} 5 \mathrm{~N} 2$ virus possessing hemagglutinin (HA) and neuraminidase (NA) genes of $\mathrm{H} 5 \mathrm{~N} 2$ virus from Mexico and internal genes of the Taiwan H6N1 virus. The reassortant $\mathrm{H} 5 \mathrm{~N} 2$ virus mutated into an HPAIV and caused outbreaks in poultry in Taiwan during 2012 (5).

In 2007, outbreaks of LPAIV H5N2 occurred among chickens in Santo Domingo and Higüey-La Otra Banda, Dominican Republic, and were reported to the World Organisation for Animal Health (OIE) (6). The OIE Reference Laboratory detected LPAIV H5N2 lineages from Mexico in samples from the Dominican Republic outbreaks on December 21, 2007. During December 2007-February 2008, a total of 11 avian influenza A viruses were detected in the Dominican Republic from backyard birds, fighting birds, and a live bird market (7) (Appendix Table 1, https://wwwnc.cdc.gov/EID/article/26/12/200268-App1.pdf). During 2007-2016, serologic surveillance of poultry detected 364/45,440 (0.80\%) samples exhibiting positive antibody responses, suggesting low level circulation of LPAIVs in the Dominican Republic, but the HA and NA subtypes were not identified (data not shown). During September-November 2017, the H5N2 LPAIV reemerged and affected 5 commercial chicken farms in Espaillat, San Juan, and La Vega (Appendix Table 2). Subsequently, the viruses were detected in 23 commercial and backyard poultry flocks during September 2018-February 2019 (8). During the 20172019 H5N2 LPAIV outbreak period, seropositivity reached $52 \%(8,740 / 16,543)$.

Since 2007, limited information on H5N2 LPAIVs and few genetic sequences have been reported. We provide sequenced genomes of 19 H5N2 LPAIVs identified in the Dominican Republic during 20072019: 1 virus from 2007, 6 from 2017, 1 from 2018, and 11 from 2019.

The 2007 H5N2 LPAIV, Ck/Dominican Republic/2007(H5N2), had an HA cleavage site sequence with 2 basic amino acids (PQRETR/G). However, the 2017-2019 viruses possessed 3 monobasic amino acids (PQRGKR/G, PQREKR/G, and LQREKR/G) (Appendix Table 3). Seven representative isolates were of low pathogenicity in chickens on intravenous inoculation (intravenous pathogenicity index $=0.0$ ). The acquisition of an additional basic amino acid in the HA cleavage site raises a concern regarding the increased risk for mutation to an HPAIV.

All genes formed a well-supported monophyletic clade (bootstrap support of 98-100 in maximum likelihood phylogeny and posterior probability of 0.99-1.00 in Bayesian phylogeny), suggesting their close relationship from a single viral introduction into the poultry population descended from A/ Ck/Hidalgo/28159-232/94 (H5N2)-like virus and maintenance in poultry in the Dominican Republic (Figure 1; Appendix Figures 1-8). The inferred time to most recent common ancestor (tMRCA) for each gene of H5N2 viruses identified in the Dominican Republic ranged from February 2005 to August 2006, suggesting that ancestors of these viruses emerged from an H5N2 virus lineage introduced from Mexico during this period (Appendix Table 4). Phylogenetic analyses show divergence of all 8 gene segments into 3 genetic sublineages 


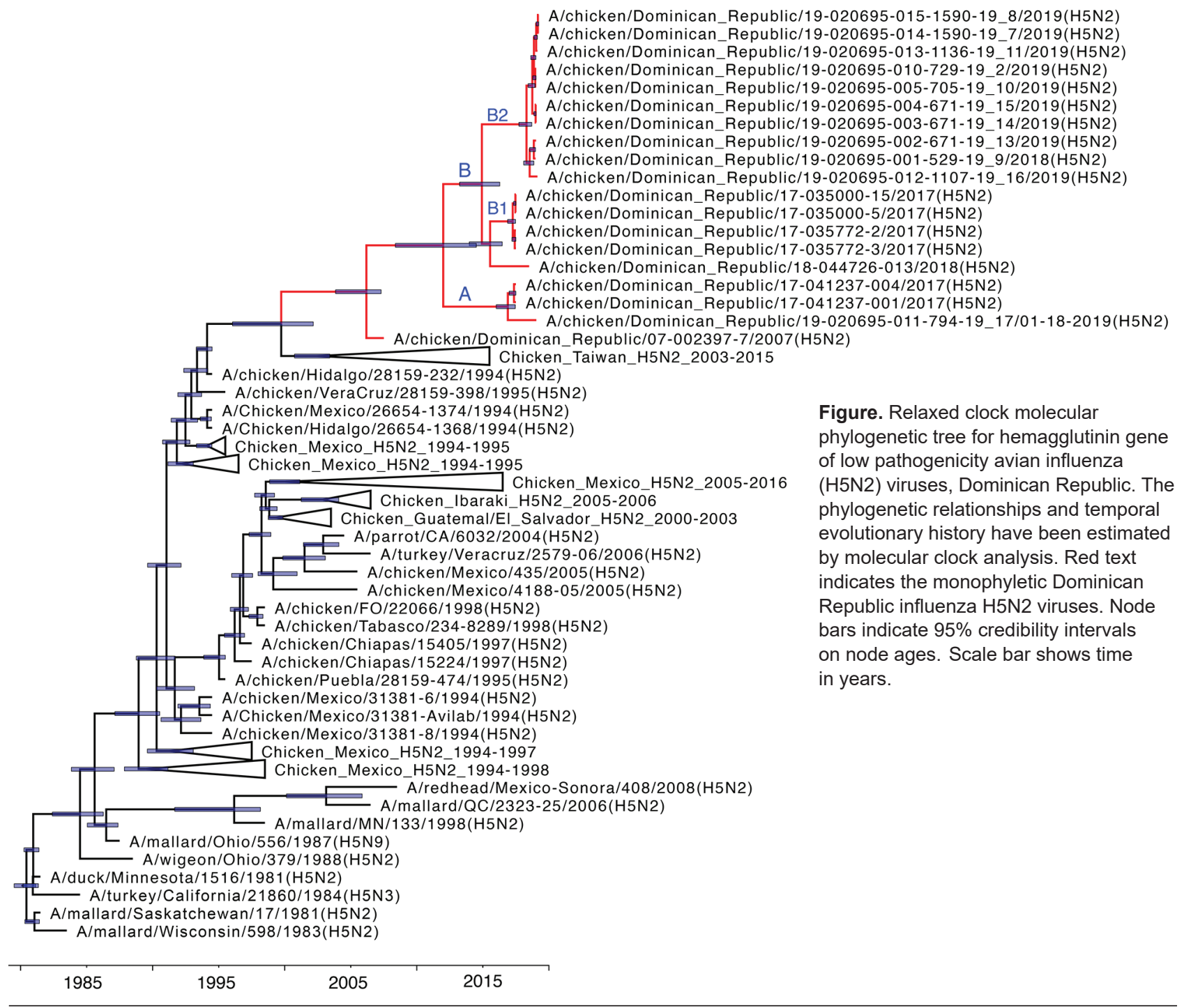

designated as sublineage $\mathrm{A}$, which contains viruses collected in 2017 and 2019; B1, which contains viruses collected during 2017; and B2, which contains viruses collected during 20182019. The 8 gene segments of $\mathrm{Ck} /$ Dominican Republic/044726_013/2018(H5N2) fell into sublineage B (Appendix Table 3).

The prediction of N-linked glycosylation sites in the HA protein revealed that the H5N2 LPAIVs have 9 potential glycosylation sites at position 27, $39,142,181,252,277,302,496$, and 555 (H5 numbering system), with a range of 6-8 sites for individual isolates (Appendix Table 5, Figure 9). The potential glycosylation at positions 142, 181, and 252 were found within antigenic sites (9). The initially identified Ck/Dominican_Republic/2007(H5N2) was predicted to contain N-linked glycosylation at the antigenic sites at positions 181 and 252. Sublineage
A and B1 did not have the predicted glycosylation at position 252 but gained additional glycosylation at position 142. However, sublineage B2 was predicted to possess glycosylation at all antigenic sites at positions 142, 181, and 252, except for the Ck/Dominican_Republic/020695-012-1107-19-16/2019 (H5N2) strain, which has $\mathrm{N}$-glycosylation only at positions 142 and 252.

Enhanced active surveillance is required to monitor the evolution and spread of H5N2 viruses in the Dominican Republic; such efforts could further the epidemiologic understanding and the design of improved prevention strategies. Additional studies could elucidate whether the genetic changes in glycosylation and antigenic sites contribute to the alterations in antigenicity of the H5N2 LPAIV from the Dominican Republic against current $\mathrm{H} 5$ virus vaccine strains. 


\section{Acknowledgment}

We thank Julia Desiato for technical assistance.

D.-H.L. and D.H.C. are partially supported by the US Department of Agriculture, Agricultural Research Service project no. 6040-32000-066-51S.

\section{About the Author}

Mr. Chung is a PhD student at the University of Connecticut, Storrs, Connecticut, USA. His primary research interests include the molecular epidemiology of avian influenza and other zoonotic viruses.

\section{References}

1. Villarreal-Chávez C, Rivera-Cruz E. An update on avian influenza in Mexico. Avian Dis. 2003;47(Suppl):1002-5. https://doi.org/10.1637/0005-2086-47.s3.1002

2. Escorcia M, Vázquez L, Méndez ST, Rodríguez-Ropón A, Lucio E, Nava GM. Avian influenza: genetic evolution under vaccination pressure. Virol J. 2008;5:15 https:// doi.org/10.1186/1743-422X-5-15

3. Abdelwhab SM, Veits J, Mettenleiter TC. Genetic changes that accompanied shifts of low pathogenic avian influenza viruses toward higher pathogenicity in poultry. Virulence. 2013;4:441-52. https://doi.org/10.4161/viru.25710

4. Bublot M, Pritchard N, Swayne DE, Selleck P, Karaca K, Suarez DL, et al. Development and use of fowlpox vectored vaccines for avian influenza. Ann N Y Acad Sci. 2006;1081:193-201. https:// doi.org/10.1196/annals.1373.023

5. Lee CC, Zhu H, Huang PY, Peng L, Chang YC, Yip CH, et al. Emergence and evolution of avian H5N2 influenza viruses in chickens in Taiwan. J Virol. 2014;88:5677-86. https:/ / doi.org/10.1128/JVI.00139-14

6. World Organization for Animal Health. Report on low pathogenic avian influenza virus in Dominican Republic, H5N2 2007. 2007 Dec 21 [cited 2019 Dec 10]. https://www.oie.int/wahis_2/public/wahid.php/ Reviewreport/Review?page_refer=MapEventSummary\&re portid $=6616$

7. World Organization for Animal Health. Report on low pathogenic avian influenza virus in Dominican Republic, H5N2 2009. 2009 Aug 21 [cited 2019 Dec 10]. https://www.oie.int/wahis_2/public/wahid.php/ Reviewreport $/$ Review? reportid $=6882$

8. World Organization for Animal Health. Report on low pathogenic avian influenza virus in Dominican Republic, H5N2 2019. 2019 Oct 16 [cited 2019 Dec 10]. https://www.oie.int/wahis_2/public/wahid.php/ Reviewreport/Review? reportid=31880

9. Kaverin NV, Rudneva IA, Govorkova EA, Timofeeva TA, Shilov AA, Kochergin-Nikitsky KS, et al. Epitope mapping of the hemagglutinin molecule of a highly pathogenic H5N1 influenza virus by using monoclonal antibodies. J Virol. 2007;81:12911-7. https://doi.org/10.1128/ JVI.01522-07

Address for correspondence: Dong-Hun Lee, Department of Pathobiology \& Veterinary Science, University of Connecticut, 61 N Eagleville Rd, Unit-3089, Storrs, CT 06269, USA; email: dong-hun.lee@uconn.edu

\section{Autochthonous Ratborne Seoul Virus Infection in Woman with Acute Kidney Injury}

\author{
Jörg Hofmann, ${ }^{1}$ Elisa Heuser, ${ }^{1}$ Sabrina Weiss, ${ }^{2}$ \\ Beate Tenner, Konrad Schoppmeyer, Jutta Esser, \\ Christiane Klier, Stephan Drewes, Rainer G. Ulrich, \\ Detlev H. Kruger
}

Author affiliations: Charité-Universitätsmedizin Berlin, Berlin, Germany (J. Hofmann, S. Weiss, B. Tenner, D.H. Kruger); Friedrich-Loeffler-Institut, Greifswald-Insel Riems, Germany (E. Heuser, S. Drewes, R.G. Ulrich); Euregio-Klinik, Medizinische Klinik II, Nordhorn, Germany (K. Schoppmeyer); Laborarztpraxis Osnabrück, Georgsmarienhütte, Germany (J. Esser); German Center for Infection Research, Partner Site Hamburg-Lübeck-Borstel-Insel Riems, Germany (E. Heuser, R.G. Ulrich); Public Health Agency of Lower Saxony, Hannover, Germany (C. Klier)

DOI: https://doi.org/10.3201/eid2612.200708

Outside Asia, Seoul virus (SEOV) is an underestimated pathogen. In Germany, autochthonous SEOV-associated hantavirus disease has not been unequivocally diagnosed. We found clinical and molecular evidence for SEOV infection in a young woman; her pet rat was the source of infection.

$\mathrm{H}$ antavirus infections cause febrile and often lifethreatening zoonoses known as hemorrhagic fever with renal syndrome and hantavirus cardiopulmonary syndrome. Human pathogenic hantavirus species usually are carried by specific rodent reservoirs, which shed infectious virus in their excreta (1).

Seoul virus (SEOV), a species within the genus Orthohantavirus, is hosted by Norway or brown rats (Rattus norvegicus) and other Rattus species as main reservoir. SEOV-associated hantavirus disease is characterized by fever, acute kidney injury, often hepatitis and gastroenteritis, associated with transient thrombocytopenia and proteinuria $(2,3)$. Most clinical cases are known to originate from China and South Korea; however, SEOV infection can occur worldwide because of the global distribution of Norway rats in the wild. Moreover, human infection has been described from contact with breeder rats (laboratory rats and laboratory rat-derived tissue cultures), pet rats, and feeder rats (3-6).

SEOV-caused hantavirus disease, especially in areas outside Asia to which it is not endemic, is

${ }^{1}$ These authors contributed equally to this article.

${ }^{2}$ Current affiliation: Robert Koch Institute, Berlin, Germany. 